\title{
Rules to Live By
}

\author{
I. Life is not fair.
}

2. What you do today will affect you tomorrow.

3. There is no such thing as a free lunch.

4. Ignorance of the law is no excuse.

5. Follow the Golden Rule: do unto others as you would have them do unto you.

6. Ask questions.

7. Never sign anything if you don't know what you are signing.

8. The more you know, the less threatened you will feel. 